\title{
Towards Prognostics of Electrolytic Capacitors
}

\author{
José R. Celaya $^{1}$ \\ SGT Inc. NASA Ames Research Center, Moffett Field, CA, 94035 \\ Chetan Kulkarni ${ }^{2}$ and Gautam Biswas ${ }^{3}$ \\ Vanderbilt University, Nashville, TN, 37235 \\ and \\ Kai Goebel ${ }^{4}$ \\ NASA Ames Research Center, Moffett Field, CA, 94035
}

\begin{abstract}
A remaining useful life prediction algorithm and degradation model for electrolytic capacitors is presented. Electrolytic capacitors are used in several applications ranging from power supplies on critical avionics equipment to power drivers for electro-mechanical actuators. These devices are known for their low reliability and given their criticality in electronics subsystems they are a good candidate for component level prognostics and health management research. Prognostics provides a way to assess remaining useful life of a capacitor based on its current state of health and its anticipated future usage and operational conditions. In particular, experimental results of an accelerated aging test under electrical stresses are presented. The capacitors used in this test form the basis for a remaining life prediction algorithm where a model of the degradation process is suggested. This preliminary remaining life prediction algorithm serves as a demonstration of how prognostics methodologies could be used for electrolytic capacitors.
\end{abstract}

\section{Nomenclature}

$\mathrm{CP} \quad=$ Pristine state measured capacitance

$E S R=$ Equivalent series resistance of the electrolytic capacitor

$E S R p \quad=$ Pristine state measured equivalent series resistance

$C_{k}=$ Measured capacitance at time $t_{k}$.

$R U L \quad=$ Remaining Useful Life

\section{Introduction}

$\mathrm{T}$ his paper proposes a model based prognostics approach for electrolytic capacitors. Electrolytic capacitors have become critical components in electronics systems in aeronautics and other domains. This type of capacitors is known for its low reliability and frequent breakdown on critical systems like power supplies of avionics equipment and electrical drivers of electro-mechanical actuators of control surfaces. The field of prognostics for electronics components is concerned with the prediction of remaining useful life (RUL) of components and systems. In particular, it focuses on condition-based health assessment by estimating the current state of health. Furthermore, it leverages the knowledge of the device physics and degradation physics to predict remaining useful life as a function of current state of health and anticipated operational and environmental conditions.

This work presents the results from an accelerated life test on electrolytic capacitors. This test applies electrical overstress to the capacitors in order to observe and record the degradation process and identify performance conditions in the neighborhood of the failure criteria in a considerably reduced time frame. An empirical degradation

\footnotetext{
${ }^{1}$ Research Scientist, Prognostics Center of Excellence and AIAA Member.

${ }^{2}$ Graduate Research Assistant, Institute for Software Integrated Systems.

${ }^{3}$ Professor, Electrical Engineering and Computer Science.

${ }^{4}$ Research Scientist, Prognostics Center of Excellence.
} 
model is presented; this model is based on the observed degradation process during the accelerated life test. A model structure is suggested based on the observed degradation curves and the model parameters are estimated using nonlinear least-squares regression. A Bayesian framework is employed to estimate (track) the state of health of the capacitor based on measurement updates of key capacitor parameters. The Kalman filter algorithm is used to track the state of health and to make a prediction of remaining useful life once no further measurements are available. A discussion and physical interpretation of the degradation model is presented. Several performance parameters are analyzed based on electro-impedance spectroscopy, like frequency response characterization via Nyquist and Bode plots, as well as parameter identification for reduced order lumped-parameter models. This analysis guides the selection of the precursor of failure variable used in the RUL prediction framework. A first order capacitance and equivalent series resistance (ESR) model is employed and the capacitance value is used in the development of the algorithm.

\section{A. Motivation}

The development of prognostics methodologies for the electronics field has become more important as more electrical systems are being used to replace traditional systems in several applications in fields like aeronautics, maritime, and automotive. The development of prognostics methods for electronics present several challenges due to great variety of components used in a system, a continuous development of new electronics technologies, and a general lack of understanding of how electronics fail. Traditional reliability techniques in electronics tend to focus on understanding the time to failure for a batch of components of the same type. Just until recently, there has been a push to understand, in more depth, how a fault progresses as a function of usage, namely, loading and environmental conditions. Furthermore, just until recently, it was believed that there were no precursor of failure indications for electronics systems. That is now understood to be incorrect, since electronics systems -similar to mechanical systems- provide early warnings to failure which could be leveraged to detect failures before they happen and to predict the remaining useful life as a function of future usage and environmental conditions

Avionics systems on-board autonomous aircrafts perform critical functions greatly escalating the ramification of an in-flight malfunction ${ }^{1,2}$. These systems combine physical processes, computational hardware, and software systems, and present unique challenges for fault diagnosis, and then establishing the effects of faults on overall system behavior. A systematic analysis of these conditions is very important for analysis of aircraft safety and also to avoid catastrophic failures during flight.

The power supplies are a critical component of modern avionics systems. Degradations and faults of the DC-DC converter unit propagate to the GPS (global positioning system) and navigation subsystems affecting the overall operation. Capacitors and MOSFETs (metal oxide field effect transistor) are the two major components, which cause degradations and failures in DC-DC converters ${ }^{3}$. Some of the more prevalent fault effects, such as a ripple voltage surge at the power supply output can cause glitches in the GPS position and velocity output, and this, in turn, if not corrected will propagate and distort the navigation solution.

Capacitors are used as filtering elements on power electronics systems. Electrical power drivers for motors require capacitors to filter the rail voltage for the H-bridges that provide bidirectional current flow to the windings of electrical motors. These capacitors help to ensure that the heavy dynamic loads generated by the motors do not perturb the upstream power distribution system. Electrical motors are an essential element in electro-mechanical actuators systems that are being used to replace pneumatic actuation in control surfaces of future generation aircrafts.

\section{B. Related work}

\section{Previous work}

In earlier work presented in Ref. 4, we studied the degradation of capacitors under nominal operation. There, work capacitors were used in a DC-DC converter and their degradation was monitored over an extended period of time. The capacitors were characterized every 100-120 hours of operation to capture degradation data for ESR and capacitance 5 . The data collected over the period of about 4500 hours of operation was then mapped against an Arrhenius inspired ESR degradation model. This is the degradation model developed for calculating the increase in the ESR over nominal degradation ${ }^{5}$.

In following experimental work, we studied accelerated degradation in capacitors ${ }^{6}$. In that experiment the capacitors were subjected to high charging/discharging cycles at a constant frequency and their degradation progress was monitored. The work presented here is the continuation of those electrical overstress (EOS) experiments and data analysis. 


\section{Other related work and current art in capacitor prognostics}

The output filter capacitor has been identified as one of the elements of a switched mode power supply that fails more frequently and have a critical impact on performance ${ }^{7,8,9}$. A prognostics and health management (PHM) approach for power supplies of avionics systems is presented in Ref. 7. Results from accelerated aging of the complete supply are presented and discussed in terms of output capacitor and power MOSFET failures; there is no modeling of the degradation process or RUL prediction for the power supply. Other approaches for prognostics for switched mode power supplies are presented in Refs. 8 and 9. The output ripple voltage and leakage current are presented as a function of time and degradation of the capacitor. No details are presented in Refs. 8 and 9 regarding the modeling of the degradation process. Furthermore, no technical details are presented in Refs. 8 and 9 in terms of fault detection and RUL prediction algorithms.

A health management approach for multilayer ceramic capacitors is presented in Ref. 10. This approach focuses on the temperature-humidity bias accelerated test to replicate failures. A method based on Mahalanobis distance is used to detect abnormalities in the test data; there is no prediction of RUL. A data driven prognostics algorithm for multilayer ceramic capacitors is presented in Ref. 11. This method uses data from accelerated aging test to detect potential failures and to make an estimation of time of failure.

\section{Accelerated aging experiments}

Accelerated life test methods are often used in prognostics research as a way to assess the effects of the degradation process through time. It also allows for the identification and study of different failure mechanisms and their relationships with different observable signals and parameters. Accelerated life test are often used in reliability studies to assess mean time to failure based on fixed operational and environmental conditions and mostly focusing in a single stressor, which could be temperature, overvoltage, humidity, etc. The work presented here is based on an accelerated test system for electrical overstress. This allows us to record several signals through the degradation process, generate run-to-failure data and experiment with different stress levels.

\section{A. Accelerated aging system description}

Since the objective of this experiment is studying the effects of high voltage on degradation of the capacitors, the capacitors were subjected to high voltage stress through an external supply source using a specially developed hardware. The capacitors are not operated within DC-DC converters; only the capacitors were subjected to the stress. Figure 1 shows the entire setup for the experiment, which includes the test board with capacitors, power supply, signal generator and the NI (National Instruments) data acquisition system.

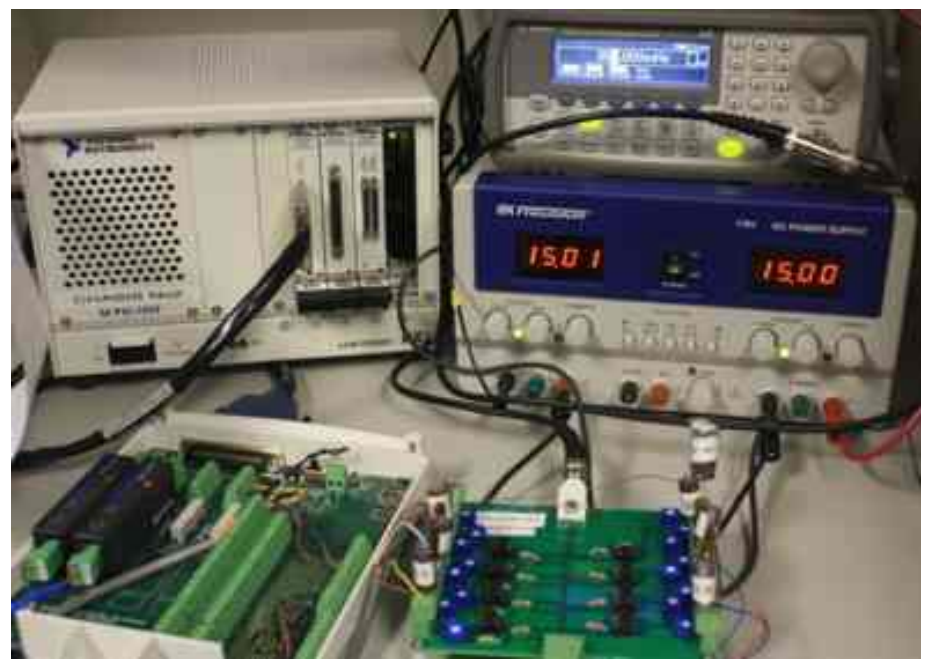

Figure 1. Hardware setup for the experiment.

A voltage source with a square wave of $200 \mathrm{mHz}$ frequency and $1 \mathrm{~V}$ output was used for generating the required signal. The output voltage from the source was then ramped up to the required voltage of $12 \mathrm{~V}$ using an external hardware circuit. This ramped up voltage was selected to be higher than the rated voltage of $10 \mathrm{~V}$ to observe accelerated degradation in the capacitor. The $200 \mathrm{mHz}$ square wave frequency output subjects the capacitor to a 
continuous charge/discharge cycle. A load of $100 \Omega$ was connected at capacitor terminal to discharge the capacitor completely within the specified cycle time.

In the charging/discharging cycle initially the capacitors charge/discharge simultaneously but as time progress and the capacitors degrades the charge/discharge time varies for each capacitor. Though all the capacitors under test are subjected to similar operating conditions their ESR and capacitance values change and this monitor this charging/discharging of each capacitor under test we measure the input and output voltages of the capacitor. Figure 2 shows the block diagram for the electrical overstress experiment.

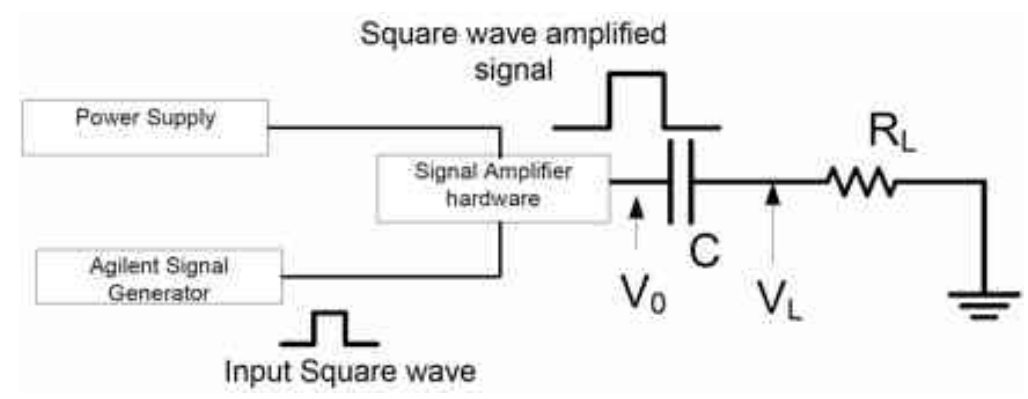

Figure 2.Block diagram of the experimental setup.

\section{B. Accelerated aging experiments on electrolytic capacitors}

For this experiment six capacitors in a set were considered for the EOS experimental setup. Electrolytic capacitors of $2200 \mu \mathrm{F}$ capacitance, with a maximum rated voltage of $10 \mathrm{~V}$, maximum current rating of $1 \mathrm{~A}$ and maximum operating temperature of $105^{\circ} \mathrm{C}$ was used for the study. These were the recommended capacitors by the manufacturer for DC-DC converters. The capacitors used for the experiments were picked from the same lot of one manufacturer, and all the capacitors in the lot had the same specifications. The electrolytic capacitors under test were characterized in detail before the start of the experiment at room temperature.

The ESR and capacitance values were measured using an SP-150 Biologic SAS measuring instrument. The average initial ESR value was measured to be around $0.056 \mathrm{~m} \Omega$ and average capacitance of $2123 \mu \mathrm{F}$ for the set of capacitors under test. ESR value is real impedance measured through the terminal software of the instrument. Similarly the capacitance value is computed from the imaginary impedance using Electrochemical Impedance Spectroscopy Z-Fit. The details of the method are presented in ${ }^{12}$.

The measurements were recorded approximately every 8-10 hours of the total 180 plus hours of operation time to capture the rapid degradation phenomenon in the ESR and capacitance values. The ambient temperature for the experiment was controlled and kept at $25^{\circ} \mathrm{C}$. During each measurement the voltage source was shut down, capacitors were discharged completely and then the characterization procedure was carried out. This was done for all the six capacitors under test. Keeping all of the conditions according to specification, the experiment was started again till the next measurement time. This procedure was followed and recorded for all the readings taken during the time of the experiment. A GUI has been developed to monitor the charge/discharge voltage using the Lab View provided by NI.

\section{Analysis of the degradation process}

There are several factors that cause electrolytic capacitors to fail. Continued degradation, i.e., gradual loss of functionality over a period of time results in the failure of the component. Complete loss of function is termed a catastrophic failure. Typically, this results in a short or open circuit in the capacitor. For capacitors, degradation results in a gradual increase in the equivalent series resistance (ESR) and decrease in capacitance over time.

The capacitor undergoes degradation when it is subjected to different operating conditions. We studied the electrical overstress operating condition for this work. The other operating conditions relate to high thermal conditions, ageing over the period of time, etc. The different physics of failure mechanisms which trigger different failure modes in a capacitor are as follows:

1.Degradation/Loss of capacitance of cathode and anode foil

2.Electrolyte evaporation

3.Degradation of the oxide film

4.Ageing/Degradation in the dielectric material 
5.Corrosion of the electrodes and

6.Increase in the internal pressure

These failure mechanism lead to different failure modes like increase in the electrical parameters above a certain threshold, increase in the leakage current, a short circuit or opening of the pressure vent or popping of the capacitor can due to increase in the internal pressure. Of all the mechanism mentioned above we discuss the ones which are related to the EOS condition in the next section. The work in Ref. 13 describes the qualitative analysis of the physics of failures under EOS conditions in detail.

\section{A. Physical interpretation of the failures due to electrical overstress}

In this work, we study the degradation of electrolytic capacitors operating under high electrical stress, i.e., $V_{\text {applied }} \geq$ $\mathrm{V}_{\text {rated. }}$ During the charging/discharging process the capacitors degrade over the period of time. A study of the literature ${ }^{14,15}$ indicated that the degradation could be primarily attributed to three phenomena:

1.Electrolyte evaporation,

2.Leakage current, and

3.Increase in internal pressure

An ideal capacitor would offer no resistance to the flow of current at its leads. However, the electrolyte, aluminum oxide, space between the plates and the electrodes produces a small equivalent internal series resistance (ESR) as shown in Figure 3a. The ESR dissipates some of the stored energy in the capacitor. In spite of the dielectric insulation layer between a capacitor's plates, a small amount of 'leakage' current flows between the plates. For a good capacitor operating nominally this current is not significant, but it becomes larger as the oxide layer degrades during operation. High electrical stress is known to accentuate the degradation of the oxide layer due to localized dielectric breakdowns on the oxide layer ${ }^{16,17}$. These breakdowns, which accelerate the degradation, have been attributed to the duty cycle, i.e., the charge/discharge cycle during operation ${ }^{16,18}$.

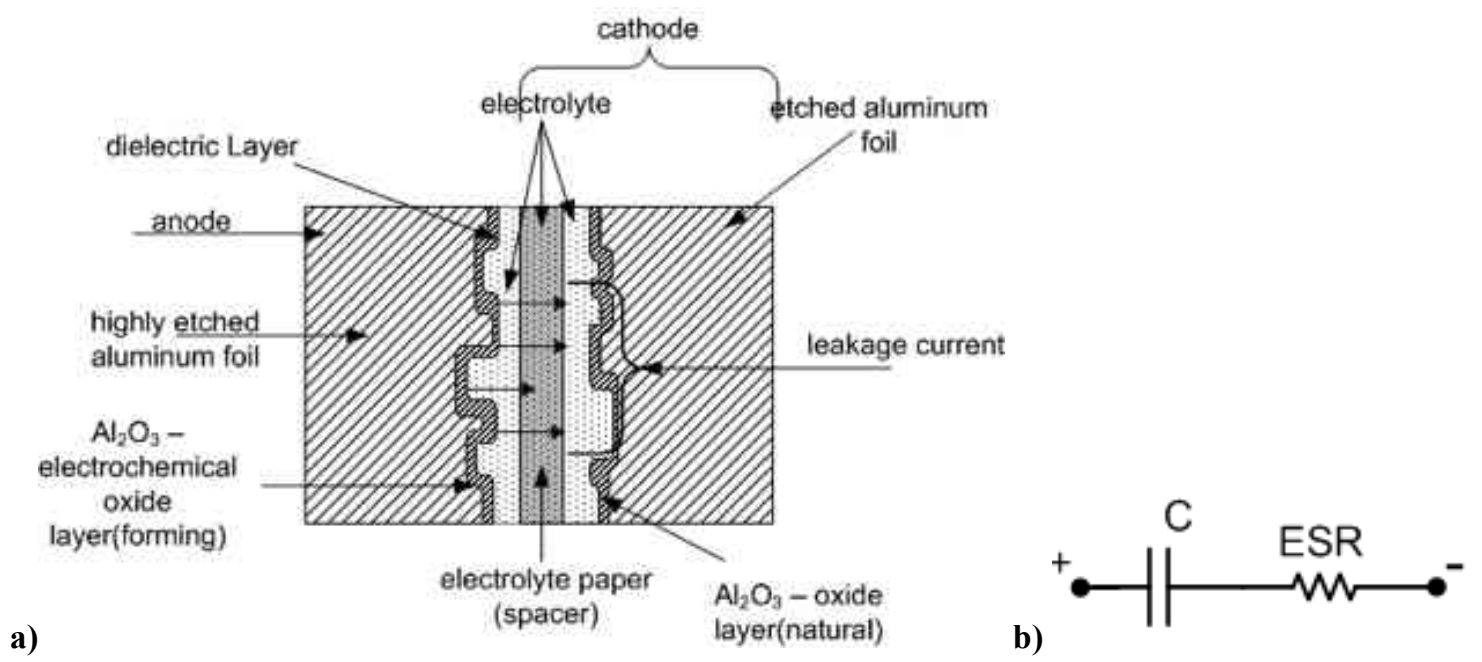

Figure 3. a) Capacitor structure and b) equivalent circuit diagram.

The literature on capacitor degradation shows a direct relationship between electrolyte decrease and increase in the ESR of the capacitor ${ }^{4}$. We have also modeled this phenomenon, and demonstrated in the effectiveness of our model through experiments in previous work ${ }^{6}$. ESR increase implies greater dissipation, and, therefore, a slow decrease in the average output voltage at the capacitor leads.Another mechanism occurring simultaneously is the increase in internal pressure due to an increased rate of chemical reactions, which are attributed to the internal temperature increase in the capacitor.

Electrolyte evaporation: Operation of the capacitors under $V_{\text {applied }} \geq V_{\text {rated }}$ results in rise of the internal core temperature of the capacitor. The current flow through the capacitor during the charge/discharge cycle has an exponential temporal relation with the applied voltage ${ }^{19}$. This has been demonstrated by a physics model we developed in previous work ${ }^{2}$. When the applied voltage, $\mathrm{V}_{\text {applied }}$ is above $\mathrm{V}_{\text {rated }}$ the rate of current flow increases at a 
much faster rate, thus increasing the amount of heat generated. Lesser amount of this heat gets dissipated to the outside, which leads to a rise in the core temperature of the capacitor. The increase in temperature accelerates electrolyte evaporation, thus increasing the ESR of the capacitor. Further details are documented in ${ }^{2}$.

Leakage Current: Changes in the electrical parameters due to degradation in the oxide layer and electrolyte depletion causes changes in the leakage current of the capacitors. Leakage current $\left(\mathrm{I}_{\mathrm{L}}\right)$ is defined as the current that flows between the capacitor plates subsequent to the charging of the capacitor ${ }^{20}$. It has been shown that the leakage current becomes more pronounced as the dielectric layer on the capacitor plates is damaged. The leakage current value is very much dependent on the effective/healthy surface area of the etched aluminum foil (capacitance of the capacitor), and the type of electrolyte used. Thus leakage currents depends of several affecting factors, such as time, voltage, temperature, type of electrolyte, and operating 'history' of the capacitor ${ }^{20}$.

Increase in Pressure: Internal pressure increases due to increase in the core temperature and chemical reactions taking place during the charge/ discharge cycle. Under normal operating conditions the chemical reactions between the dielectric and electrolyte releases hydrogen gas, which is then absorbed by the anode and cathode surfaces. The absorption of the gas keeps the pressure inside the capacitor from increasing too much. However, as the internal temperature increases the chemical reaction rate increases producing more hydrogen, but the absorption rate is unable to keep up. The excess hydrogen gas, which is generated, causes the internal pressure to increase ${ }^{21}$. This pressure increase can ultimately lead to the capacitor popping.

\section{B. Failure precursors}

During the experiments as discussed earlier we were also characterizing the capacitors at regular intervals. ESR and capacitance are the two main failure precursors that identify the current health state of the device. The schematic diagram reflects these two parameters as and ESR in series with the capacitor shown in Figure: $3 \mathrm{~b}$. ESR and capacitance values were calculated after characterizing the capacitors. As the devices degrade due to different failure mechanisms we can observe a decrease in the capacitance and an increase in the ESR

Figure 4 describes the plot for degradation in the ESR and capacitance. Figure 4a shows percentage increase in the ESR value for all the six capacitors under test over the period of time. This value of ESR is calculated from the impedance measurements after characterizing the capacitors. Similarly, figure $4 \mathrm{~b}$ shows the percentage decrease in the value of the capacitance as the capacitor degrades over the period under EOS test condition discussed. As per the standards MIL-C- $62 \mathrm{~F}^{14}$ a capacitor is considered unhealthy if under electrical operation its ESR increases by 280 $300 \%$ of its initial value or the capacitance decreases by $20 \%$ below its pristine condition value. From the plots we observe that for the time for which the experiments were conducted the average ESR value increases by almost $54 \%-55 \%$ while over the same period of time average capacitance decreased by more than $20 \%$ that is the threshold mark for a healthy capacitor.
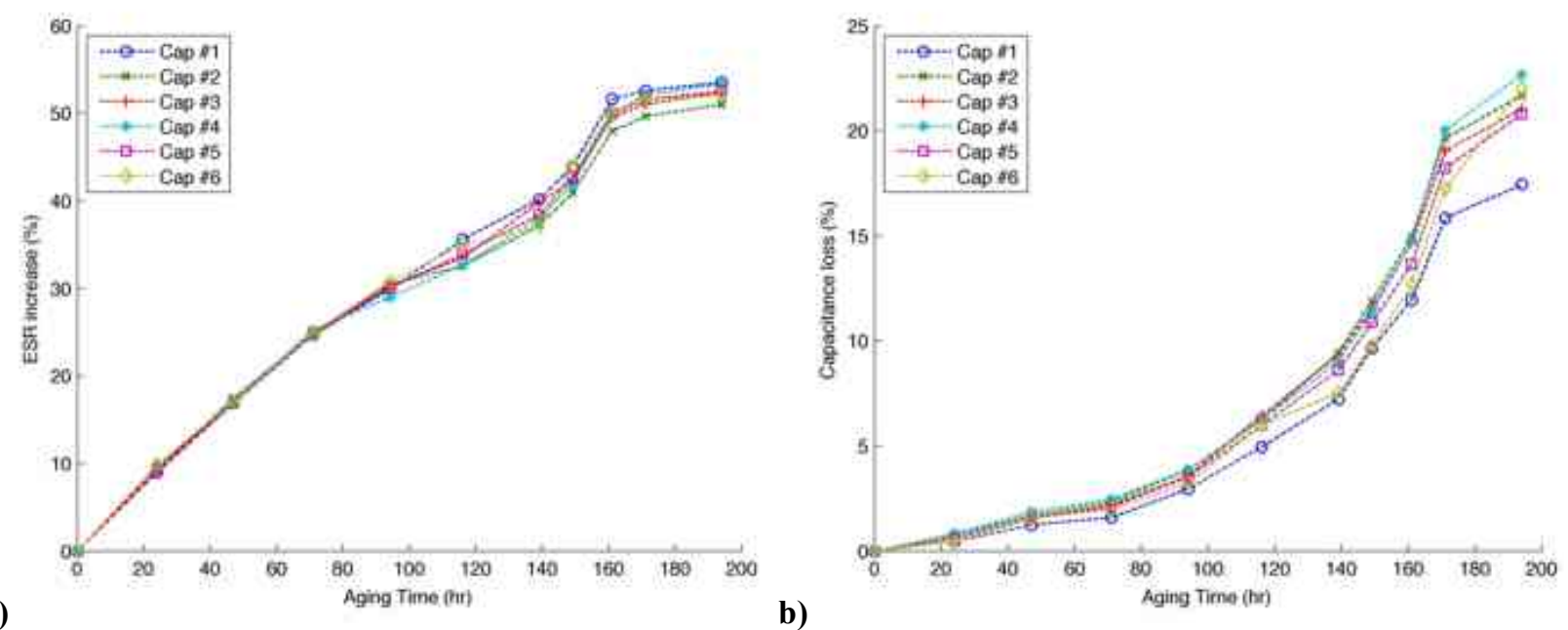

Figure 4.Degradation of capacitor performance: a) percentage ESR increase as a function of aging time, b) percentage capacitance loss as a function of aging time. 
As mentioned earlier, the characterizations of the capacitors were done at regular time intervals. In this section, we discuss the data plots with respect to degradation of the device. We can capture the impedance data as a Nyquist data plot as shown in the figure 5 below (Nyquist plot for the capacitor at different time intervals of operation). From the plots we observe that the capacitor had minimum impedance measured at pristine condition, which increases as the device degrades over time due to the electrical overstress.

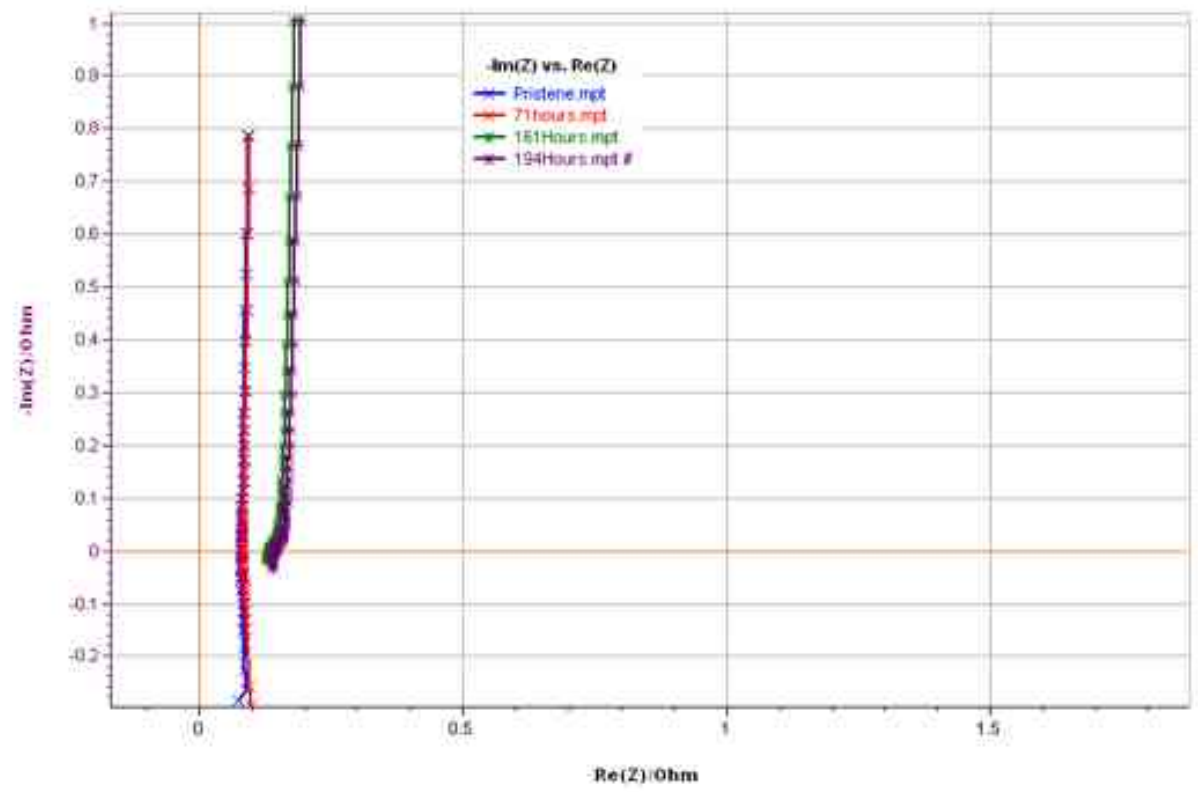

Figure 5.Overall Nyquist Plot for the impedance measurements.

Once can observe a significant degradation mechanism as indicated by the curvy nature in the degraded capacitors.

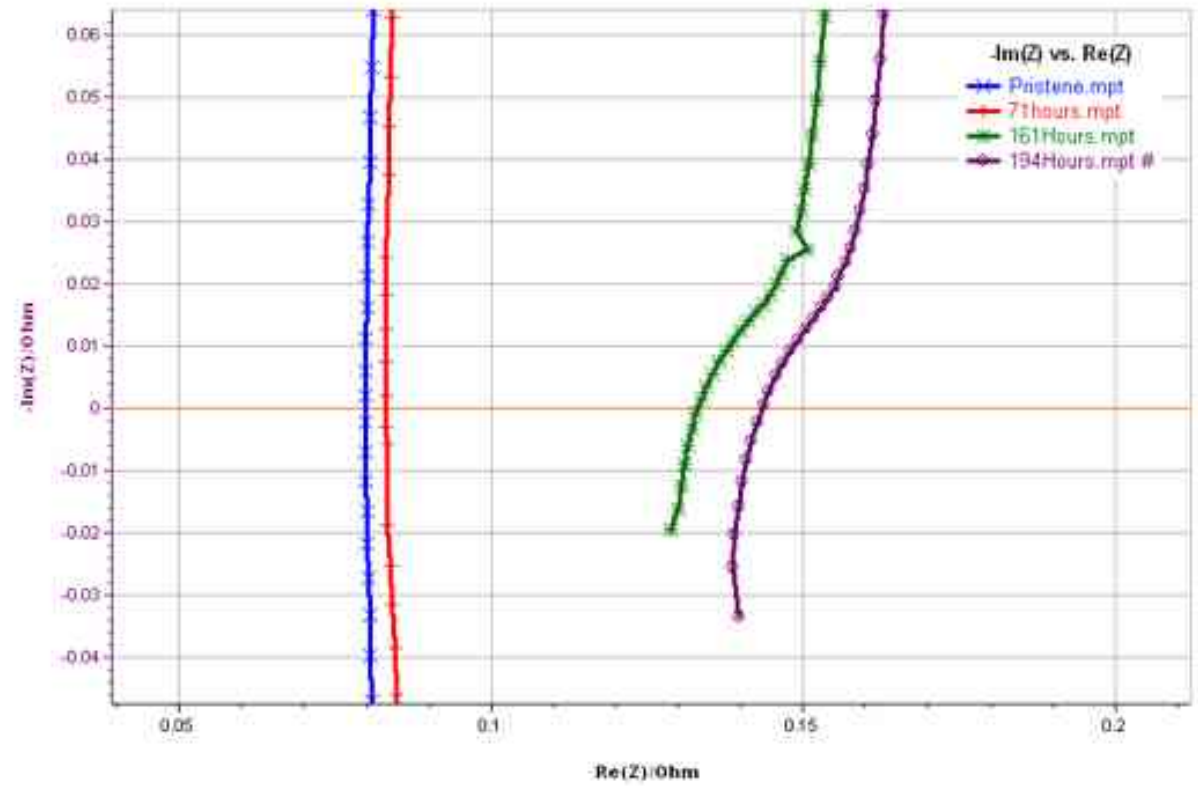

Figure 6.Detailed Nyquist Plot for the impedance measurements.

We hypothesize that this behavior is related to the formation of the crystallization layer due to voltage sparking on the surface of the oxide. In a damage model, this behavior can be reproduced as a small capacitance with 
resistance in parallel along with the exiting ESR and capacitance. The equivalent circuit diagram can be modeled as shown in figure 7.

For the same data we also plotted the Bode plot (figure 8), which shows the frequency response relating to the changes in the capacitance of the device at different operating times measured. It can be observed that as the capacitor degrades the magnitude decreases indicating the decrease in capacitance of the device. Earlier in the section we discussed a decrease in the capacitance value due to increase in the internal temperature. This can be shown by the bode plot in figure 8 .

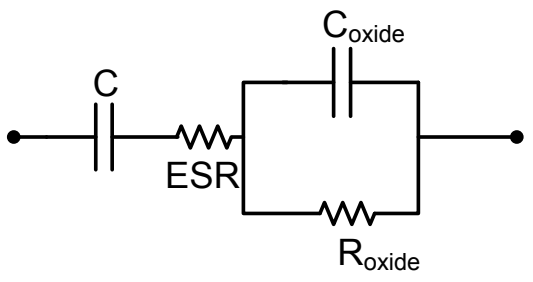

Figure 7.Updated degradation model of the capacitor.

The other part of the Bode plot describes how the phase is changing as the capacitance decreases and the ESR increases. Under normal operating conditions the phase angle is high, almost towards $90^{\circ}$ (a perfect capacitor will have a phase angle of $90^{\circ}$ ). As the capacitance starts decreasing due to degradation we observe decrease in the phase angle value. This is also an indication of the device's degradation as a result of decrease in capacitance and increase in ESR.

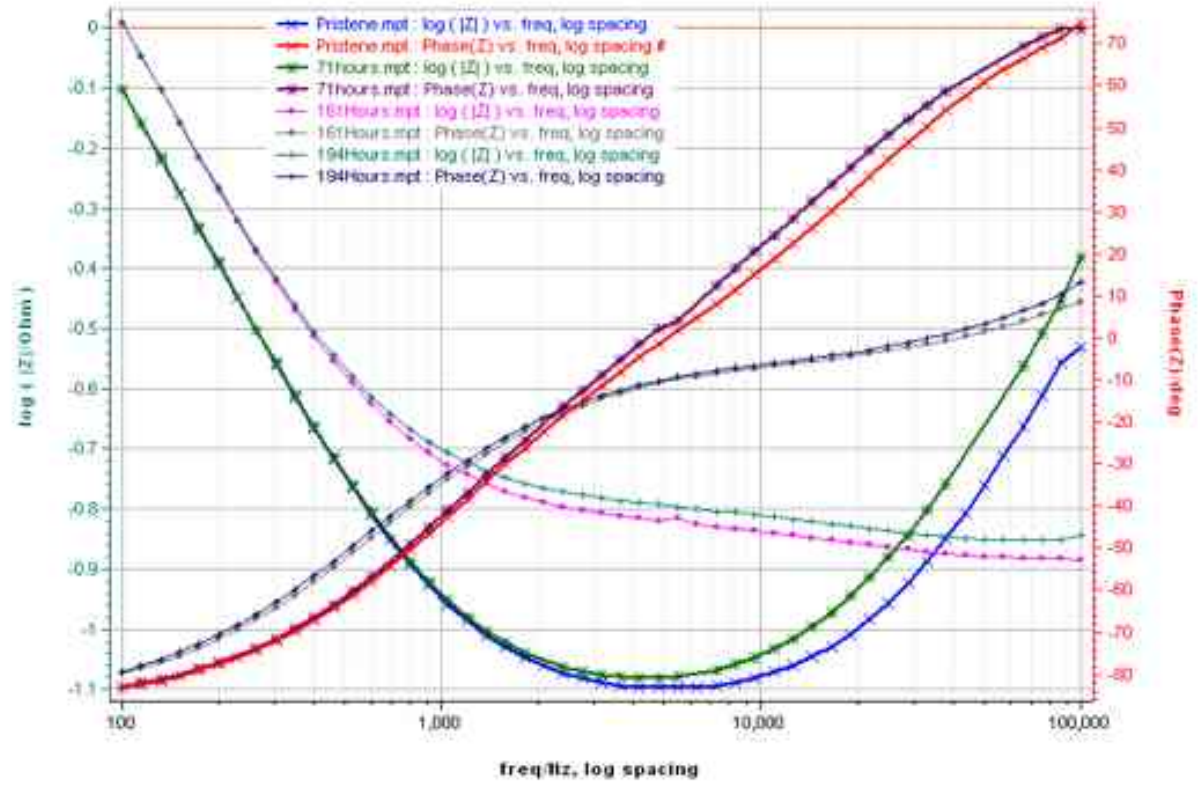

Figure 8.Bode Plot for the impedance measurements.

\section{Prediction of Remaining Useful Life}

A model-based prognostics algorithm based on Kalman filter and a physics inspired empirical degradation model is presented. This algorithm is able to predict remaining useful life of the capacitor based on the accelerated degradation data from the experiments described in previous sections. The percentage loss in capacitance is used as a precursor of failure variable and it is used to build a model of the degradation process. This model relates aging time to the percentage loss in capacitance and has the following form,

$$
C_{k}=e^{\alpha t_{k}}+\beta
$$


where $\alpha$ and $\beta$ are model constants that will be estimated from the experimental data of accelerated aging experiments. In order to estimate the model parameters, five capacitors are used for estimation (\#1-\#5), and the remaining capacitor (\#6) is used to test the prognostics algorithm. A nonlinear least-squares regression algorithm is used to estimate the model parameters. Figure 9 shows the estimation results. The experimental data is presented together with results from the exponential fit function. It can be observed from the residuals that the estimation error increases with time. This is to be expected since the last data point measured for all the capacitors fall slightly off the concave exponential model. The estimated parameters are $\alpha=0.0163$ and $\beta=-0.5653$.
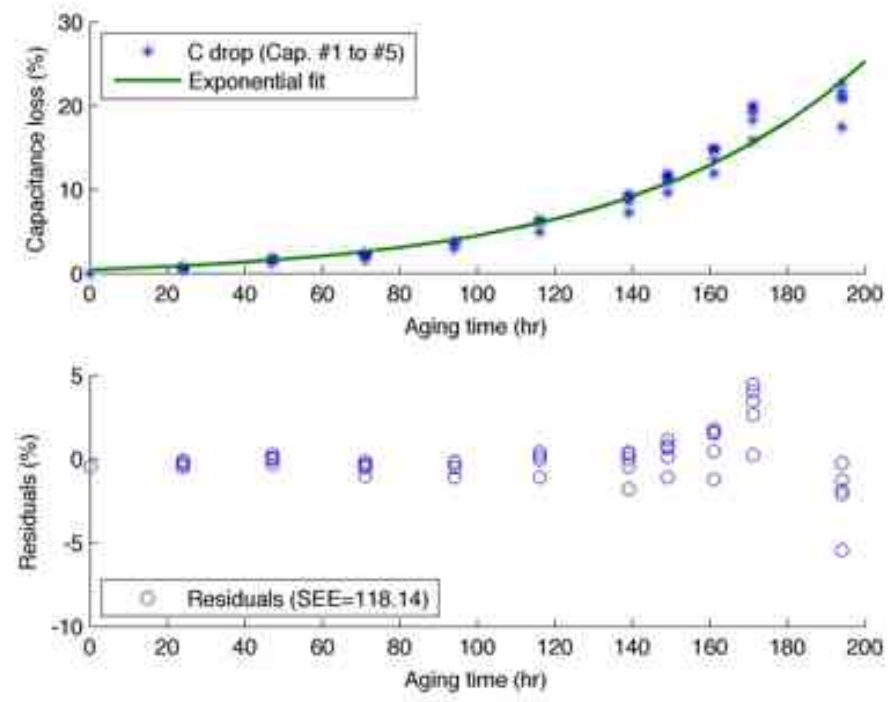

Figure 9.Estimation results for the empirical degradation model.

The estimated degradation model is used as part of a Bayesian tracking framework to be implemented using the Kalman filter technique. This method requires a state-space dynamic model relating the degradation level at time $t_{k}$ to the degradation level at time $t_{k-1}$. The formulation of the state model is described below.

$$
\begin{gathered}
\frac{\partial C}{\partial t}=\alpha C-\alpha \beta \\
\frac{C_{t}-C_{t-\Delta t}}{\Delta t}=\alpha C_{t-\Delta t}-\alpha \beta \\
C_{t}=\left(1+\alpha \Delta_{t}\right) C_{t-\Delta t}-\alpha \beta \Delta_{t} \\
C_{k}=(1+\alpha \Delta k) C_{k-1}-\alpha \beta \Delta_{k}
\end{gathered}
$$

In this model $C_{k}$ is the state variable and it represents the percentage loss in capacitance. Since the system measurements are percentage loss in capacitance as well, the output equation is given by $y_{k}=h C_{k}$, where the value of $h$ is equal to one. The following system structure is used in the implementation of the filtering and the prediction using the Kalman filter.

$$
\begin{gathered}
C_{k}=A_{k} C_{k-1}+B_{k} u+v \text { and } y_{k}=h C_{k}+w, \text { where } \\
A_{k}=\left(1+\Delta_{t}\right), \\
B_{k}=-\alpha \beta \Delta_{k}, \\
h=1, u=1 .
\end{gathered}
$$


The time increment between measurements $\Delta_{k}$ is not constant since measurements were taken at non-uniform sampling rate. This implies that some of the parameters of the model in equation 3 will change through time. Furthermore, $v$ and $w$ are normal random variables with zero mean and $Q$ and $R$ variance respectively. The description of the Kalman filtering algorithm is omitted from this article. A thorough description of the algorithm can be found in Ref. 22, a description of how the algorithm is used for forecasting can be found in Ref. 23 and an example of its usage for prognostics can be found in Ref. 24. Figure 10 shows the results of the application of the Kalman filter to the test case (Cap. \#6). The model noise variance $Q$ was estimated from the model regression residuals. The residuals have a mean very close to zero and a variance of 2.1829. This variance was used for the model noise in the Kalman filter implementation. The measurement noise variance $R$ is also required in the filter implementation. This variance was computed from the direct measurements of the capacitance with the electroimpedance spectroscopy equipment, the observed variance is $4.99 \mathrm{E}-7$. Figure 10 shows the result of the filter tracking the complete degradation signal. The residuals show an increased error with aging time. This is to be expected given the results observed from the model estimation process.
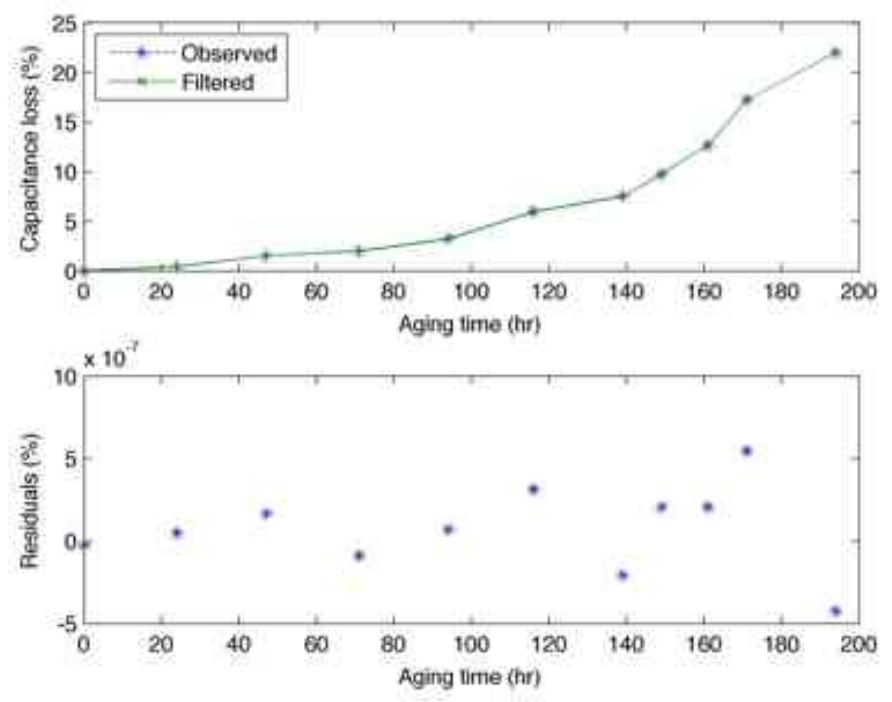

Figure 10.Tracking results for the Kalman filter implementation applied to test capacitor (\#6).

The use of the Kalman filter as a forecasting algorithm requires the evolution of the state without updating the error covariance matrix and the posterior of the state vector. The $n$ step ahead forecasting equation for the Kalman filter is given below. The last update is done at the time of the last measurement $t_{l}$.

$$
\hat{C}_{l+n}=A^{n} C_{l}+\sum_{i=0}^{n-1} A^{i} B
$$

The subscripts from parameters $A$ and $B$ are omitted since a constant $\Delta_{t}$ is used in the forecasting mode (one prediction every hour). Figure 11 presents results from the remaining useful life prediction algorithm at time 149 (hr), which is the time at which an ESR and $C$ measurements are taken. The failure threshold is considered to be a crisp value of $20 \%$ decrease in capacitance. End of life (EOL) is defined as the time at which the forecasted percentage capacity loss trajectory crosses the EOL threshold. Therefore, RUL is EOL minus 149 hours. 


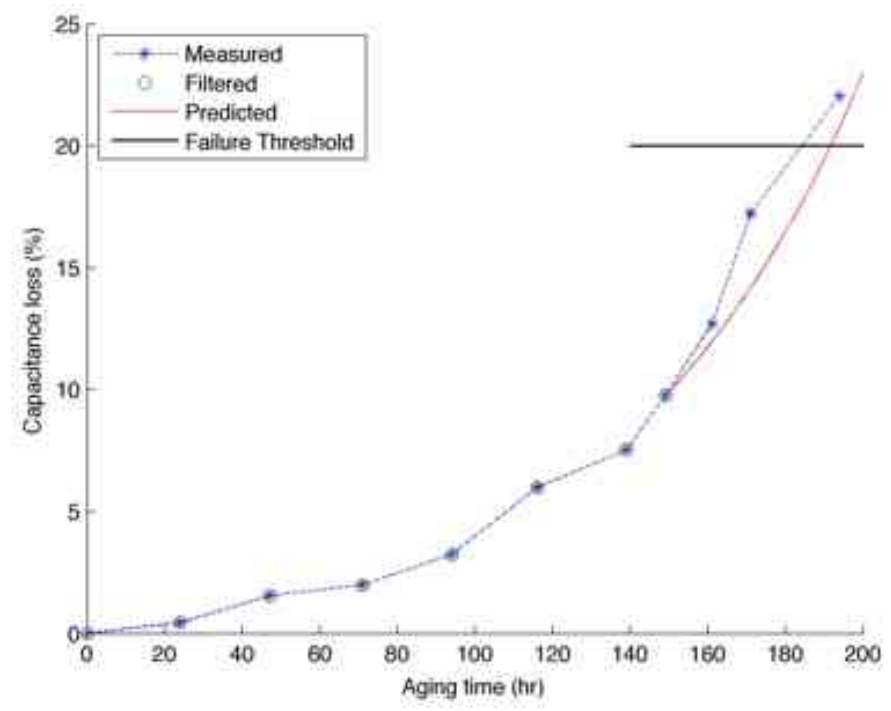

Figure 11.Remaining useful life prediction at time 149 (hr).

Table 1 summarizes results for the remaining life prediction at all points in time where measurements are available.

Table 1.Summary of RUL forecasting results.

\begin{tabular}{|c|l|l|}
\hline $\begin{array}{c}\text { RUL } \\
\text { forecasting } \\
\text { time (hr) }\end{array}$ & $\begin{array}{l}\text { RUL } \\
\text { estimate } \\
\text { (hr) }\end{array}$ & $\begin{array}{l}\text { Ground } \\
\text { truth (hr) }\end{array}$ \\
\hline 0 & 222.2 & 184.24 \\
\hline 24 & 186.55 & 160.24 \\
\hline 47 & 140.66 & 137.24 \\
\hline 71 & 128.98 & 113.24 \\
\hline 94 & 104.18 & 90.24 \\
\hline 116 & 70.71 & 68.24 \\
\hline 139 & 57.58 & 45.24 \\
\hline 149 & 42.61 & 35.24 \\
\hline 161 & 27.20 & 23.24 \\
\hline 171 & 8.94 & 13.24 \\
\hline
\end{tabular}

\section{Conclusion}

This paper presents a RUL prediction algorithm based on accelerated life test data and an empirical degradation model. The main contributions of this work are: a) the identification of the lumped-parameter model (figure $3 b$ ) for a real capacitor as a viable reduced-order model for prognostics-algorithm development; $b$ ) the identification of ESR and $\mathrm{C}$ (from $\mathrm{ESR}+\mathrm{C}$ ) model as precursor of failure feature parameters; c) the development of an empirical degradation model based on accelerated life test data which accounts for shifts in capacitance as a function of time; d) the implementation of a Bayesian based health state tracking and remaining useful life prediction algorithm based on the Kalman filtering framework. One major contribution of this work is the prediction of remaining useful life for capacitors as new measurements become available.

Results of the remaining useful life prediction open an avenue for further research and developments and increase in the technology readiness level of prognostics applied to electrolytic capacitors. The results presented here are based on accelerated life test data and on the accelerated life timescale. Further research will focus on development of functional mappings that will translate the accelerated life timescale into real usage conditions time-

11

American Institute of Aeronautics and Astronautics 
scale, where the degradation process dynamics will be slower, and subject to several types of stresses. The performance of the proposed exponential-based degradation model is satisfactory for this study based on the quality of the model fit to the experimental data and the RUL prediction performance as compared to ground truth. As part of future work we will also focus on the exploration of additional models based on the physics of the degradation process. Additional experiments are currently underway to increase the number of test samples. This will greatly enhance the quality of the model, and guide the exploration of additional degradation-models, where the loading conditions and the environmental conditions are also accounted for towards degradation dynamics.

\section{Acknowledgments}

This work was funded by the NASA Aviation Safety Program, IVHM project and NRA grant \# NNX07ADIZA. The authors would like to express their gratitude to intern Ruperto Solis (Chihuahua Institute of Technology, México) for helping with the accelerated aging experiments and electro-impedance spectroscopy characterization.

\section{References}

${ }^{1}$ Bhatti, U., and Ochieng, W. "Failure modes and models for integrated GPS/INS systems," The Journal of Navigation Vol. 60,2007 , p. 327

${ }^{2}$ Kulkarni, C., Biswas, G., and Koutsoukos, X. "A prognosis case study for electrolytic capacitor degradation in DC-DC converters," International Conference on Prognostics and Health Management ( PHM 2009) Vol. PHMC_09_56, 2009.

${ }^{3}$ Kulkarni, C., Biswas, G., Bharadwaj, R., and Kim, K. "Effects of Degradation in DC-DC Converters on Avionics Systems: A Model Based Approach," Machinery Failure Prevention Technology Conference, MFPT 2010, 2010, pp. 8-13.

${ }^{4}$ Kulkarni, C., Biswas, G., Koutsoukos, X., Goebel, K., and Celaya, J. "Physics of Failure Models for Capacitor Degradation in DC-DC Converters," The Maintenance and Reliability Conference, MARCON 2010, 2010.

${ }^{5}$ Kulkarni, C., Biswas, G., Koutsoukos, X., Goebel, K., and Celaya, J. "Experimental Studies of Ageing in Electrolytic Capacitors," Annual Conference of the Prognostics and Health Management Society, 2010.

${ }^{6}$ Kulkarni, C., Biswas, G., Koutsoukos, X., Celaya, J., and Goebel, K. "Integrated diagnostic/prognostic experimental setup for capacitor degradation and health monitoring," AUTOTESTCON, 2010 IEEE. 2010, pp. 1-7.

${ }^{7}$ Orsagh, R., Brown, D., Roemer, M., Dabnev, T., and Hess, A. "Prognostic health management for avionics system power supplies," Aerospace Conference, 2005 IEEE. 2005, pp. 3585-3591.

${ }^{8}$ Judkins, J. B., Hofmeister, J., and Vohnout, S. "A Prognostic Sensor for Voltage Regulated Switch-Mode Power Supplies," Aerospace Conference, 2007 IEEE. 2007, pp. 1-8.

${ }^{9}$ Goodman, D., Hofmeister, J., and Judkins, J. "Electronic prognostics for switched mode power supplies," Microelectronics Reliability Vol. 47, No. 12, 2007, pp. 1902-1906.

${ }^{10}$ Nie, L., Azarian, M. H., Keimasi, M., and Pecht, M. "Prognostics of ceramic capacitor temperature-humidity-bias reliability using Mahalanobis distance analysis," Circuit World Vol. 33, No. 3, 2007, pp. 21 - 28.

${ }^{11} \mathrm{Gu}$, J., Azarian, M. H., and Pecht, M. G. "Failure prognostics of multilayer ceramic capacitors in temperature-humidity-bias conditions," Prognostics and Health Management, 2008. PHM 2008. International Conference on. 2008, pp. 1-7.

${ }^{12}$ Biologic. "Application note 14-Zfit and equivalent electrical circuits." 2010.

${ }^{13}$ Kulkarni, C. S., Biswas, G., Celaya, J., and Goebel, K. "Prognostic Techniques for Capacitor Degradation and Health Monitoring," The Maintenance \& Reliability Conference, MARCON 2011. Knoxville, TN, 2011.

${ }^{14}$ MIL-C-62F. "General Specification For Capacitors, Fixed, Electrolytic." 2008.

${ }^{15}$ IEC. "60384-4-1 Fixed capacitors for use in electronic equipment." 2007-03.

${ }^{16}$ Ikonopisov, S. "Theory of electrical breakdown during formation of barrier anodic films," Electrochimica Acta Vol. 22, 1977, pp. 1077-1082.

${ }^{17}$ Wit, H. J. D., and Crevecoeur, C. "The dielectric breakdown of anodic aluminum oxide," Physics Letters A Vol. December, 1974, pp. 365-366.

${ }^{18}$ Gomez-Aleixandre, C., Albella, J. M., and Martinez-Duarte, J. M. "Pressure build-up in aluminum electrolytic capacitors under stressed voltage conditions," Journal of Applied Electrochemistry Vol. 16, No. 1, 1986, pp. 109-115.

${ }^{19}$ Alwitt, R. S., and Parler, S. G. "Heat generation during pulse operation of prototype aluminum electrolytic capacitors," Journal of Applied Electrochemistry Vol. 25, No. 6, 1995, pp. 533-542-542.

${ }^{20}$ Roederstein, V. "Aluminum Capacitors- General Information - 25001." 2007.

${ }^{21}$ Gomez-Aleixandre, C., Albella, J. M., and Martinez-Duarte, J. M. "Gas Evolution in Aluminum Electrolytic Capacitors," J. Electrochem. Soc Vol. 131-3, 1984, pp. 612-614.

${ }^{22}$ Stengel, R. F. Optimal Control and Estimation: Dover Books on Advanced Mathematics, 1994.

${ }^{23}$ Chatfield, C. The Analysis of Time Series: An Introduction: Chapman \& Hall/CRC, 2003.

${ }^{24}$ B. Saha, K. G., and Christophersen, J. "Comparison of Prognostic Algorithms for Estimating Remaining Useful Life of Batteries," Transactions of the Institute of Measurement \& Control Vol. 31, No. 3-4, 2009, pp. 293-308. 